\title{
Small-scale Materials Behavior from $X$-ray Microdiffraction and Imaging
}

\author{
Rozaliya I. Barabash and Gene E. Ice
}

This JOM topic highlights novel applications of x-ray microdiffraction and imaging to study structural properties of materials at a small scale. The development of ultra-brilliant synchrotron X-ray sources provides important new opportunities for the analysis of local and near-surface material structures. This topic includes manuscripts that describe how different versions of polychromatic and monochromatic microdiffraction can be used to study small scale plasticity in a range of materials. A variety of ingenious methods are described as developed in laboratories around the world.

W. Ludwig et al. present an approach to crystal structure characterization based on monochromatic beam diffraction and imaging. They have developed diffraction contrast tomography as a means of studying the threedimensional (3-D) crystal structure of polycrystalline samples. This method uses changes in the direct-beam attenuation due to diffraction and tomographic methods to reconstruct the local crystal structure of polycrystalline materials.

The manuscript by O.M. Barabash et al. demonstrates the combined application of $\mathrm{x}$-ray synchrotron polychromatic and monochromatic 3-D differential aperture $\mathrm{x}$-ray microscopy (3D DAXM) in a reflection geometry for the analysis of depth-dependent dislocation densities and lattice rotations around an indented nickel-based superalloy. Polychromatic 3D DAXM allows the mapping of local lattice rotations and geometrically necessary dislocation evolution with depth in different zones of the indented nickelbased superalloy. Monochromatic 3D DAXM is particularly sensitive to the depth-dependent elastic lattice strain

\section{The development of}

\section{ultra-brilliant}

synchrotron x-ray

sources provides

\section{important new}

opportunities for the

analysis of local and

near-surface material

\section{structures.}

distribution and to the total dislocation density including statistically stored and geometrically necessary dislocations. With this method a region of residual compression is observed in the vicinity of the indent. An elasto-plastic transition zone is found to occur at approximately $20 \mu \mathrm{m}$ from the outside edge of the indent.

Ralph Nyilas et al. and the group from ETH Zurich describe two sets of in-situ synchrotron experiments. One is conducted at Swiss Light Source
(SLS) and another at the Advanced Light Source (ALS) in Berkeley, California. The microdiffraction experiments at the SLS use a transmission geometry to make in-situ uniaxial tensile measurements on thin gold films. The experiments at ALS use a reflection geometry and describe an in-situ study under biaxial strains during temperature cycling.

Helena Van Swygenhoven et al. and the group from Paul Scherre Institute in Switzerland describe Laue microdiffraction transmission-geometry experiments. This geometry is well-suited for in-situ studies of compression tests on micropillars. The authors show that there are two deformation regimes in pillars focus ion beamed from large single crystals. Initially deformation starts with activity on a slip system which differs from those predicted for uniaxial compression; the second regime corresponds to the highest Schmid factor for uniaxial compression. Experimental results are compared to the simulations.

Several other examples of x-ray diffraction techniques to study small scale will be presented in upcoming issues.

Rozaliya I. Barabash, research professor, and Gene E. Ice, Oak Ridge National Laboratory corporate fellow, are with Oak Ridge National Laboratory in Tennessee. Dr. Barabash is also with the Materials Science and Engineering Department, University of Tennessee, Knoxville, TN 37996 and can be reached at barabashr@ornl.gov. Dr. Barabash is the guest editor from the SMD Mechanical Behavior of Materials Committee.

\section{Rozaliya I. Barabash and Gene E. Ice are TMS Members!}

To read more about them, turn to page 9. To join TMS, visit www.tms.org/Society/Membership.aspx. 